\title{
Bio-Nanomedicine for Cancer Therapy Preface
}

\section{Fontana, Flavia}

Springer International Publishing AG

2021

Fontana , F \& Santos , H A 2021 , Bio-Nanomedicine for Cancer Therapy Preface . in F Fontana \& HA Santos (eds), BIO-NANOMEDICINE FOR CANCER THERAPY . Advances in Experimental Medicine and Biology, vol. 1295 , Springer International Publishing AG , Cham , pp. V-VI . https://doi.org/10.1007/978-3-030-58174-9

http://hdl.handle.net/10138/332932

https://doi.org/10.1007/978-3-030-58174-9

Downloaded from Helda, University of Helsinki institutional repository.

This is an electronic reprint of the original article.

This reprint may differ from the original in pagination and typographic detail.

Please cite the original version. 


\section{Preface}

Cancer still represents a heavy societal burden with profound economic implications. Tumors are treated with chemotherapeutic drug molecules with challenging properties for their formulation and unspecific total body toxicity when administered systemically. The application of nanotechnology to medicine, particularly for cancer treatment, has provided physicians with novel weapons in the fight against the diseases. Nanotechnology refers to the development and use of systems characterized by sizes of $1-1000 \mathrm{~nm}$. The use of nanotechnology in cancer treatment has brought along a revolution in the treatment of patients, enabling therapeutic paradigm shifts and ameliorating patients' quality of life. In nanomedicine, the small particles are loaded with anti-cancer drugs, altering their biodistribution compared to the free drug. This research field combines the expertise of engineers and material scientists, pharmacists, physicians, and immunologists to obtain carefully optimized nanoparticles to improve the drug treatment efficacy, while lowering the drug's side effects. The field of nanomedicine draws several parallels from "cooking" where an optimized "recipe" can produce "complex flavored dishes."

Nanotechnology has been, and is currently, playing a major role in cancer treatment, with several formulations for the delivery of chemotherapeutics and multiple others as companion diagnostics. Some of the conventional drug delivery nanosystems have been approved more than 20 years ago, profoundly affecting the treatment options. These nanosystems have also paved the way for research and the application of nanomedicines in other therapeutic indications, such as anesthetics, treatment of iron deficiency, antifungine delivery, and vaccines. The latest approvals have focused on the delivery of nucleic acids for gene therapy, such as in Onpattro $^{\circledR}$, and the recent developed COVID-19 vaccines. Conventional nanoparticles, however, in most cases only reduce or ameliorate a drug's side effect profile without affecting the efficacy of the drug. Interestingly, the combination of conventional nano-delivery systems with hyperbaric oxygen treatment greatly enhances the therapeutic efficacy.

Conventional nanoparticles are also lacking a complete zip-code, they are immitted in the circulation and the postal system hope they will find the right target. Peptides can serve as exquisite address labels, facilitating the delivery of drug-loaded nanosystems at the tumor. Alternatively, the combination of cells with nanoparticles in the biohybrid systems modifies the circulation pathway of the nanoparticles by mimicking the natural constituents of the human body. The labelling of these systems with radioactive tags can help in visualizing their biodistribution with potential therapeutic applications in radiotherapy.

Once at the tumor site, particular characteristics of the nanosystems, such as high porosity, and the presence of metals can provide multiple mode of action for the nanocarrier. Together with carrying drugs, they can carry large biomolecules like enzymes or react with lasers to increase the tumor temperature.

Sometimes, however, nanoparticles are not the best choice for all the applications in cancer treatment and can be exchanged for mats of electrospun nanofibers. Nanoneedles represent another alternative particularly for sampling and intracellular drug delivery to specific cell targets.

Research in cancer nanomedicine has resulted into thousands of promising therapies. However, the efficacy has to be evaluated in suitable in vitro models, with the possibility to design automated systems for high-throughput screening. 3D models, and spheroids in par- 
ticular, are currently considered the state-of-art as in vitro tumor models. Moving further in the development pipeline, solid and translatable in vivo tumor models are highly needed to provide reliable information before starting the clinical trials.

Finally, cancer treatment has been overturned by the discoveries and applications of immunotherapy. In some of the patients, immunotherapy has achieved a complete cure. Nanotechnology plays a role with cancer nanovaccines, providing the immune system with a more powerful response. Moreover, the perfect example of nanosystems is viruses. Viruses have been shaped by millennia of co-evolution with the human body and can be modified to infect and destroy only cancer cells, while establishing a massive immune activation, either alone or combined with other inorganic/organic systems (biohybrid materials).

In this book, we include chapters explaining the basics of nanomedicine, together with the most recent research in cancer immunotherapy. This book is unique in containing both elements to provide the reader with solid basis before moving to the following developments. In this respect, the present book represents a guide on cancer nanotechnology, written by outstanding specialists in the field, for an advanced and specialist readership, while being relevant for the wider readers in academia and in private companies. Moreover, this book is designed with a thought towards the younger generations of under- and post-graduate students, to provide a 101 guide in the world of cancer nanomedicine. Thus, we collected informative and valuable contributions from top-level researchers in material science, pharmacy, biology, radiochemistry, and immunology, working together on the development of advanced nanomedicines/nanoparticles for cancer treatment applications. Overall, this book provides a highly valuable support for the readers in the field of cancer nanomedicine.

We would like to express our gratitude to all the authors and contributors of this book for their cooperation and enthusiasm in writing the book chapters, spacing from classical nanosystems to nanoneedles, to radioactive probes, and in vitro and in vivo models for the screening of nanoparticles, as well as to the latest developments in biohybrid nanoparticles and immunotherapy with cancer vaccines and oncolytic viruses, and the industrial and commercial perspective on nanotechnology in cancer immunotherapy. The concepts presented in each chapter are clarified by selected and explicative examples. We wish the readers will enjoy the contents of the book and hope that this book will promote discussion about the role of nanotechnology in cancer therapy.

Helsinki, Finland

Flavia Fontana Hélder A. Santos 